\title{
Croyances philosophiques et droit des discriminations
}

Jo Carby-Hall

\section{(2) OpenEdition}

1 Journals

Édition électronique

URL : https://journals.openedition.org/rdctss/758

DOI : $10.4000 /$ rdctss.758

ISSN : 2262-9815

Traduction(s) :

The evolutionary tract of philosophical beliefs constituting protected characteristics in British

Discrimination Law - URL : https://journals.openedition.org/rdctss/2780 [en]

Éditeur

Centre de droit comparé du travail et de la sécurité sociale

Édition imprimée

Date de publication : 1 avril 2021

Pagination : 194-199

ISSN : 2117-4350

Référence électronique

Jo Carby-Hall, "Croyances philosophiques et droit des discriminations », Revue de droit comparé du travail et de la sécurité sociale [En ligne], 1 | 2021, mis en ligne le 01 avril 2021, consulté le 02 avril 2022. URL : http://journals.openedition.org/rdctss/758; DOI : https://doi.org/10.4000/rdctss.758

\section{(c) (i) (9)}

Revue de droit comparé du travail et de la sécurité sociale est mise à disposition selon les termes de la Licence Creative Commons Attribution - Pas d'Utilisation Commerciale - Pas de Modification 4.0 International. 


\section{JO CARBY-HALL}

UNIVERSITÉ DE HULL

\section{CROYANCES PHILOSOPHIQUES ET DROIT DES DISCRIMINATIONS}

Ces dernières années la jurisprudence relative aux croyances philosophiques a connu une importante évolution et constitue un des développements les plus intéressants du domaine du droit des discriminations au Royaume-Uni. En vertu de l'Equality Act de $2020^{1}$, neufs " motifs protégés » ${ }^{2}$ des discriminations ont été identifiés ${ }^{3}$. On ne traitera ici que des croyances philosophiques qui ont connu l'évolution la plus significative.

L'Equality Act de 2010 ne définit pas l'expression «croyance philosophique ». Il dispose ${ }^{4}$ que "croyance » recouvre toute croyance religieuse ou philosophique, renvoyant donc au juge le soin de définir la notion. Lord Nicholls ${ }^{5}$ a posé les bases d'une définition dans ces termes : " une croyance doit remplir un minimum raisonnable de critères et d'objectifs (...), elle doit être cohérente avec les notions de dignité humaine et d'intégrité (...), ne doit pas se rapporter à des choses insignifiantes. Elle doit être d'un niveau de sérieux et d'une importance suffisante (...), elle doit s'attacher à un problème fondamental, être cohérente c'est-à-dire intelligible et facile à comprendre (...), sans être excessivement exigeant à cet égard. Dans l'ensemble, les critères de base ne doivent pas être fixés à un niveau si élevé que cela empêcherait les croyances minoritaires de bénéficier de la protection dont elles devraient jouir $»$.

Dans l'affaire Grainger Plc v Nicholson'6, Mr Justice Burton, adoptant le dictum de Lord Nicholls dans l'affaire Williamsom, tenta d'établir cinq critères pour définir l'expression «croyance philosophique $»^{7}:$ : (i) la croyance doit être authentique ; (ii) il faut que ce soit une croyance et non pas une opinion ou un point de vue basé sur des informations actuellement disponibles; (iii) cette croyance doit se rapporter à un aspect essentiel de la vie et du comportement humain ; (iv) elle doit avoir un certain niveau de puissance, de sérieux, de cohésion et d'importance ; (v) elle doit être digne de respect dans une société démocratique, ne pas enfreindre les critères de dignité humaine ni les droits d'autrui ».

5 Dans l'affaire $R$ Williamson et al $v$ Secretary of State for Education and Employment, even though he suffered discrimination on grounds of his beliefs. [2005] AC248 UK HL 15, paragraphe 23 du jugement. 
Bien que de toutes les affaires n'aient pas répondu aux critères ainsi posés ${ }^{8}$, celles qui seront ci-après analysées ont abouti. Notons d'ores et déjà que toutes ces affaires traitent de problèmes autres que la croyance philosophique; elles concernent les licenciements économiques, abusifs ou injustifiés.

Dans l'affaire Grainger susmentionnée, la croyance au changement climatique causé par l'homme, avec les impératifs moraux qui en découlent, peut, si elle est authentique, être considérée comme croyance philosophique.

Les faits sont les suivants. M. Grainger, qui était chargé du développement durable au sein de la compagnie, fut licencié. II argua que ses convictions en matière de changement climatique avaient contribué à son licenciement, ce qui était discriminatoire car il n'avait fait qu'exprimer sa position sur le sujet : il n'était pas d'accord avec la politique de la compagnie sur les émissions de gaz à effet de serre. Ses supérieurs hiérarchiques entravaient ses fonctions en lui refusant l'accès aux données qui lui auraient permis de calculer l'empreinte carbone de la compagnie. En outre, il relatait que les cadres se déplaçaient dans des voitures extrêmement polluantes et que le PDG, qui avait oublié son smartphone à l'étranger, avait envoyé un de ses salariés le rechercher en avion.

L'Employment Appeal Tribunal confirma le verdict de l'Employment Tribunal : la position du requérant remplissait les critères d'une croyance philosophique.

Dans l'affaire D.Maisiry $v$ British Broadcasting Corporation", la croyance du requérant se fondait sur la notion de "service public ", lequel serait préservé des ingérences du gouvernement, de l'État et des intérêts économiques. Selon lui, c'est grâce au service public qu'un individu peut faire l'expérience des échanges culturels, de l'éducation et de débats, et c'est là qu'il peut partager ses expériences. Des philosophes tels que Jurgen Habermas et Dewey ont mis en évidence le sens, la cohérence, l'importance et le sérieux de cette interprétation du service public. Le Juge estima que, vu le passé du requérant, les quatre critères de Nicholson étaient remplis.

De même, concernant la chasse au renard et l'abattage des lièvres, le fait de croire au caractère sacré de la vie fut jugé comme étant une croyance philosophique dans l'affaire Hasham v Milton Park (Dorset) Ltd t/a Orchard Park ${ }^{10}$. Le requérant vivait et travaillait pour la protection des animaux contre la maltraitance. Hormis pour son habillement et son alimentation, ses convictions impactaient son travail,

8 Par exemple : l'importance de ne pas arborer un coquelicot le 11 novembre (Lisk) ; I'homosexualité en contradiction à la loi divine, la négation de l'holocauste (Ellis) ; l'affirmation que les attentats terroristes des 9/11 et 7/7 étaient une conspiration du gouvernement (Farrel); le refus aux partenaires de même sexe de pouvoir adopter ou d'être une famille d'accueil (McClintock); le refus de signature d'un accord sur les droits d'auteur (Gray); le port d'une croix en argent en contravention au règlement de British Airways (Eweida); comme il n'y a que deux sexes, le sexe est biologique et immuable (Foestater) ; le végétalisme n'est pas une croyance philosophique (Conisbee); le politiquement correct (Dunn); le refus d'identifier ses patients transgenres par leur sexe choisi au cours d'une visite médicale (Mackereth).

9 Case $n^{\circ} \mathrm{ET} / 1313142 / 2010$.

10 Case $n^{\circ} \mathrm{ET} / 3105555 / 2009$. 
déterminaient les lieux qu'il fréquentait et la manière dont il utilisait son temps et ses ressources.

Le Juge estima que la preuve de la croyance était crédible et remplissait les cinq critères établis en jurisprudence ; il conclut que les croyances philosophiques du requérant méritaient la protection de la législation.

Le républicanisme en tant que croyance philosophique a été retenu dans l'affaire Gibbins $v$ British Council11. La requérante croyait fermement que le Royaume-Uni ne devait pas être régi par une monarchie héréditaire et devait être une République démocratique et laïque. Cette conviction s'était manifestée progressivement à travers sa participation à des manifestations antiracistes ou dénonçant les circonstances de la mort de Blair Peach; au contact et sous l'influence de Toni Benn - républicain convaincu dont elle avait lu les écrits - ; à travers son appartenance syndicale, au Parti Travailliste, à Amnesty International, etc. Athée et socialiste, elle était connue comme «The Quiet Corbinista ${ }^{12}$.

Le Juge exprima la position suivante: "Nous pensons que les croyances de la requérante lui permettent de s'identifier à une opposition à l'héritage et aux privilèges que représente la monarchie (...) et à un système de gouvernance par une élite aristocratique. Ceci concorde avec les idées de la gauche sur d'autres aspects de l'organisation sociale. C'est aussi une croyance de poids et essentielle sur notre mode de gouvernement. C'est à notre avis une croyance philosophique qui garantit à cette personne la protection contre la discrimination ».

Dans I'affaire C. Oliver $v$ Department of Work and Pensions ${ }^{13}$, le requérant affirmait que le Parti Travailliste n'est pas uniquement un groupement, qu'il incarne un ensemble de croyances connues sous le vocable de socialisme démocratique et qu'il en est garant.

Le juge considéra les cinq critères relatifs à la « croyance philosophique » et conclut: « Je trouve que ceci équivaut à une croyance et non pas juste à une opinion ou un point de vue (...) et que c'est une croyance en un aspect de la vie et du comportement humain qui est substantiel et a du poids et qui a un certain niveau de puissance, de sérieux, de cohésion et d'importance. II s'en suit que les critères de " croyance philosophique " sont bien remplis. Je conclus que la croyance en un socialisme démocratique, qui anime le requérant ${ }^{14}$, tombe sous la définition de l'expression «croyance philosophique » et suffit pour jouir de la protection de la section 10 de l'Equality Act 2010 ».

Dans l'affaire A Power v Greater Manchester Police Authority ${ }^{14}$, M. Alan Power, "spiritualiste convaincu », croyait sincèrement et de bonne foi en l'existence du « spiritualisme psychique » et aux phénomènes paranormaux. L'Employment

11 Case $n^{\circ} E T / 2200088 / 2017$.

12 En référence à l'ex-leader du Parti Travailliste dont les vues étaient d'extrême gauche.

13 Case ${ }^{\circ} E T / 701407 / 2013$.

14 [2010 UKEAT 0087/10 and [201] EWCA CV. 534, jugé par Stanley Burton L. J. 
Tribunal jugea ${ }^{15}$ que les croyances spiritualistes et psychiques, la croyance en Dieu et en la vie éternelle, pouvaient constituer une croyance religieuse philosophique au titre de la législation. Le juge retraça l'histoire de l'Eglise Spiritualiste et considéra que la croyance du requérant en la vie éternelle et en la possibilité de communiquer avec les esprits méritait le respect dans une société démocratique, et remplissait les critères de cohésion, de puissance, de sérieux et d'importance requis pour être une croyance philosophique.

Dans Hawkins v United Utilities Ltd t/a Unicorn ${ }^{16}$, le jugement fut basé sur une supposition. Le requérant avait été licencié pour ne pas avoir atteint les objectifs fixés. Celui-ci affirma que le motif réel de son licenciement était lié à ses croyances chrétiennes qui lui interdisaient de mentir. II allégua qu'on lui avait conseillé de mentir et qu'il avait surpris certains de ses collègues qui mentaient au téléphone à des clients potentiels et les bernaient ainsi. Le requérant perdit son procès car il n'avait pas apporté de preuves suffisantes pour appuyer sa requête. Néanmoins, l'Employment Tribunal affirma qu'une authentique croyance, selon laquelle il ne faut pas mentir pour tromper des clients potentiels et les convaincre de faire un achat, aurait été jugée comme une croyance philosophique au titre de la section 10 de I'Equality Act si les cinq critères établis par l'affaire Nicholson avaient été remplis.

Le sens de la responsabilité dans les services publics peut être qualifié de croyance philosophique. Dans l'affaire J Anderson v Chesterfield High School ${ }^{17}$, le requérant, inspiré par ses parents et un activiste local, croyait fermement qu'il devait servir la communauté pour le bien de tous. Cette conviction le conduisit à s'investir dans le conseil municipal dont il devient un des membres à Liverpool. II prit du service dans le conseil d'administration de Liverpool Vision en charge du développement économique de la ville. Comme maire, il apporta sa contribution à l'intérêt commun, y compris par le retour des paquebots de croisière dans cette ville, la Fondation Oliver King, son opposition à la taxe sur les chambres (Bedroom tax) et par bien d'autres initiatives encore.

Dans l'affaire $M$ Harron v Chief Constable of Dorset Police ${ }^{18}$, le requérant croyait fermement que les services publics gaspillaient les fonds publics, et se crut obligé d'exprimer sa croyance malgré la discrimination à laquelle il s'exposait. L'intervention de l'Employment Appeal Tribunal permit à sa croyance d'être reconnue comme croyance philosophique.

Dans l'affaire C McEleny v Ministry of Defence ${ }^{19}$, I'Employment Tribunal jugea que le plaignant, qui croyait fermement à l'indépendance de l'Ecosse, défendait une croyance philosophique au titre de la section 10 de l'Equality Act de 2010,

15 La décision de l'Employment Tribunal en ce qui concerne la croyance philosophique du requérant fut confirmée par l'Employment Appeal Tribunal et la Cour d'Appel, en même temps que d'autres points soulevés dans cette affaire.

16 Case $n^{\circ} \mathrm{ET} / 2501234 / 2012$.

17 Case $n^{\circ}$ UKEAT/2006/14/MC.

18 Case n'UKEAT/0234/15/DA.

19 Case nET4105347/2017, date du jugement 9 février 2019, car c'était une affaire complexe qui posait d'autres problèmes. 
sur laquelle il s'appuyait comme caractéristique protégée pour sa requête en discrimination.

Le Juge ${ }^{20}$ énonça : «Sur les bases des éléments présentés, (...) je trouve que le requérant croit fermement que l'Ecosse devrait être indépendante, (...) qu'il soutient le SNP dont la principale ligne politique est l'indépendance (...), qu'il croit depuis son enfance que les écossais ont le droit à l'autodétermination (...), que le résultat du référendum, avec 1,5 million de voix favorables, montre qu'il n'est pas le seul dans ce cas, (...) tout cela constitue la preuve qu'il détient une croyance philosophique ».

Le requérant dans l'affaire J. Casamitjana Costa $v$ The League Against Crual Sports ${ }^{21}$, non seulement suivait un régime alimentaire végétalien, mais encore s'opposait à l'utilisation et l'exploitation des animaux dans quelque but que ce soit. Son végétalisme éthique dominait tous les aspects de sa vie. Le tribunal décida que, dans ce cas, le végétalisme éthique méritait protection en tant que croyance philosophique car le requérant menait sa vie d'une manière que beaucoup jugeraient extrême.

Dans cette affaire, le facteur déterminant fut la force des croyances du requérant et leur omniprésence dans tous les domaines de sa vie. Par cette décision, le tribunal n'indique en aucune façon une volonté de protéger tous les travailleurs végétaliens, ni même une majorité d'entre eux, mais seulement ceux dont les points de vue et actions correspondent au niveau le plus élevé de végétalisme éthique. II sera trs rare qu'un employeur découvre un tel degré d'adhésion chez ses travailleurs. C'est ce que l'on peut du reste dire de toutes les affaires similaires relatives aux croyances philosophiques.

Dans cette sélection d'affaires la croyance philosophique a été prouvée comme dans bien d'autres qui ne seront pas ici exposées. Les affaires traduites en justice pour croyance philosophique peuvent être complexes. Le requérant doit fournir la preuve et convaincre la Cour que sa croyance peut être qualifiée de croyance philosophique remplissant les critères de la section 10 de l'Equality Act de 2010.

Quand le requérant a prouvé les faits, il appartient au tribunal de juger si ceux-ci remplissent les cinq critères retenus pour la définition légale établie par M. Justice Burton dans l'affaire Nicholson. Le concept de croyance philosophique est l'un des aspects les plus intéressants du développement du droit des discriminations. Les tribunaux mettent l'accent sur l'importance des faits dans chaque cas d'espèce pour déterminer la validité de la croyance philosophique. Ceci pourrait signifier que le nombre de croyances philosophiques protégées est plus limité que ce que l'on pourrait penser au regard des exemples cités, car bon nombre de requérants risquent de ne pas prouver le degré de conviction requis. Etablir une croyance philosophique digne de la protection du droit des discriminations n'est qu'un premier pas. L'Employment Judge Hughes l'a illustré dans l'affaire Maistry en expliquant que «le véritable champ de bataille surgit quand il s'agit de déterminer si

$20 \mathrm{lbid}$. Paragraphes 4-6 du jugement.

21 Case $n^{\circ} E T / 333129 / 2018$, audience le 1 er janvier 2020. 


\section{ROYAUME-UNI}

le plaignant a été traité de manière moins favorable et, si c'est le cas, de déterminer si sa croyance en est la cause». 